\title{
Some New Aspects of Rational Interpolation
}

\author{
By Claus Schneider and Wilhelm Werner
}

\begin{abstract}
A new algorithm for rational interpolation based on the barycentric formula is developed; the barycentric representation of the rational interpolation function possesses various advantages in comparison with other representations such as continued fractions: it provides, e.g., information concerning the existence and location of poles of the interpolant.
\end{abstract}

1. Introduction. Let $r: \mathbf{R} \rightarrow \mathbf{R}$ be the rational function defined by

$$
r(t):=\frac{\sum_{i=0}^{n} \alpha_{i} f_{i} /\left(t-t_{i}\right)}{\sum_{i=0}^{n} \alpha_{i} /\left(t-t_{i}\right)} \quad\left(\text { if } t \neq t_{i}, i=0(1) n\right) .
$$

Obviously, $\lim _{t \rightarrow t_{i}} r(t)=f_{i}$ if $\alpha_{i} \neq 0$; thus $r$ is a rational interpolant of the data $\left(t_{i}, f_{i}\right), i=0(1) n$, if $\alpha_{i} \neq 0, i=0(1) n$, and $t_{i} \neq t_{j}$ for $i \neq j$. In order to fix the constants $\alpha_{i}, i=0(1) n$, one can impose additional conditions on $r$. If we set

$$
p(t)=\sum_{i=0}^{n} \alpha_{i} f_{i} \prod_{j=0 ; j \neq i}^{n}\left(t-t_{j}\right), \quad q(t)=\sum_{i=0}^{n} \alpha_{i} \prod_{j=0 ; j \neq i}^{n}\left(t-t_{j}\right),
$$

then $r=p / q$. Usually, the following assumptions are made:

$$
p \in \Pi_{m}, \quad q \in \Pi_{k}, \quad m+k=n, m \geqslant k ;
$$

here $\Pi_{j}$ denotes the space of real polynomials of degree $\leqslant j$. (As is well known, one can treat the case $m<k$ by interpolation of the data $\left(t_{i}, 1 / f_{i}\right), i=0(1) n$, such that the degree of the numerator is $k$ and the degree of the corresponding denominator is $m$; some simple modifications must be made if some of the $f_{i}$ 's vanish.) Note that our point of view differs from the usual one: since our ansatz (1) already ensures the interpolation property (if $\alpha_{i} \neq 0, i=0(1) n$, at least) we can add any reasonable conditions on $p$ and $q$, for example (2). The standard approach is to prescribe the form of $p$ and $q$; the coefficients of $p$ and $q$ are then determined such that $p$ interpolates $f q$ at $t_{0}, t_{1}, \ldots, t_{n}$.

If $q=1$, then (1) represents the barycentric form of the Lagrangian interpolation polynomial; hence the coefficients $\alpha_{i}, i=0(1) n$, of (1) can be determined with the same number of operations which is necessary to compute the Newtonian form of the interpolation polynomial via divided differences (cf. [16]).

The barycentric representation of rational interpolants has a very remarkable property: Even if the coefficients $\alpha_{i}$ are disturbed, for example, by round off, the exact interpolation property still holds (if the disturbed $\alpha_{i}, i=0(1) n$, do not vanish,

Received October 5, 1984; revised May 3, 1985 and October 10, 1985.

1980 Mathematics Subject Classification. Primary 65D05, 65D25.

(1986 American Mathematical Society $0025-5718 / 86 \$ 1.00+\$ .25$ per page 
at least). Our analogue (1), (2) of the Lagrangian interpolation polynomial differs from others (e.g., due to Cauchy [1], Salzer [10], Predonzan [9]) since our representation does not reflect the actual degree of $p$ and $q$. It must be emphasized, however, that the advantages of our method, which will be discussed in detail later, are essentially based on the representation of $r$ : They are lost if $r$ is represented in a different way.

Our method is reliable, i.e., it produces a solution of the interpolation problem if such a solution exists; otherwise, a rational interpolant for the attainable points is constructed and the unattainable points are detected. In contrast to other existing methods, our device also produces information concerning the existence and location of poles of $r$.

Our paper is organized as follows: Section 2 contains background material; some of the results presented are not new, but we give-for the reader's convenience-new proofs in our terminology. The announced algorithms for rational interpolation are detailed in Section 3; in Section 4 we derive some differentiation formulas based on rational interpolation.

2. Basic Facts on Rational Interpolation. In the sequel, we denote by $f\left[t_{0}, \ldots, t_{l}\right]$ the $l$ th divided difference of a function $f$ with respect to the nodes $t_{i}, i=0(1) l$. We will frequently use the following fact:

Lemma 1. Let $j \in\{0,1, \ldots, l\}$ and $g_{j}(t):=t-t_{j}$. Then $\left(f g_{j}\right)\left[t_{0}, \ldots, t_{l}\right]=$ $f\left[t_{0}, \ldots, t_{j-1}, t_{j+1}, \ldots, t_{l}\right]$.

With $q \in \Pi_{k}$ we associate the unique polynomial $p \in \Pi_{m}$ which interpolates $f q$ at $t_{0}, \ldots, t_{m} ;$ thus

$$
(f q)(t)=p(t)+\prod_{j=0}^{m}\left(t-t_{j}\right)(f q)\left[t_{0}, \ldots, t_{m}, t\right] .
$$

This relation plays a central role in the following considerations; as a consequence of (3) we get

Proposition 2. Assume that $q \in \Pi_{k}$ and that $p \in \Pi_{m}$ interpolates fq at $t_{0}, \ldots, t_{m}$, where $m+k=n, m \geqslant k$. Then the following statements are equivalent:

(4) $p$ interpolates fq at $t_{0}, \ldots, t_{n}$.

(5) $(f q)\left[t_{0}, \ldots, t_{m}, t_{i}\right]=0, i=m+1(1) n$.

(6) $(f q Q)\left[t_{0}, \ldots, t_{n}\right]=0$ for any $Q \in \Pi_{n-\operatorname{deg}(p)-1}$.

(7) Let l be such that $\operatorname{deg}(p)<l \leqslant n$; then for any subset $\left\{s_{0}, \ldots, s_{l}\right\}$ of $\left\{t_{0}, \ldots, t_{n}\right\}$ with cardinality $l+1:(f q)\left[s_{0}, \ldots, s_{l}\right]=0$.

Proof. (4) $\Rightarrow$ (5): Set $t:=t_{i}, i=m+1(1) n$, in (3).

(5) $\Rightarrow(6)$ : Set $\nu:=\operatorname{deg}(p)$; then $p \in \Pi_{\nu}$ and

$$
p(t)=\sum_{i=0}^{m}(f q)\left[t_{0}, \ldots, t_{i}\right] \prod_{j=0}^{i-1}\left(t-t_{j}\right)
$$

implies

$$
(f q)\left[t_{0}, \ldots, t_{i}\right]=0, \quad i=\nu+1(1) m .
$$


The functions $\omega_{i}, i=\nu+1(1) n$,

$$
\omega_{i}(t):= \begin{cases}\prod_{j=i+1}^{n}\left(t-t_{j}\right) & \text { if } \nu+1 \leqslant i \leqslant m, \\ \prod_{\substack{j=m+1 \\ j \neq i}}^{n}\left(t-t_{j}\right) & \text { if } m+1 \leqslant i \leqslant n,\end{cases}
$$

obviously form a basis of $\Pi_{n-\nu-1}$; repeated application of Lemma 1 shows that (5), (8) are equivalent to the equations

$$
\left(f q \omega_{i}\right)\left[t_{0}, \ldots, t_{n}\right]=0, \quad i=\nu+1(1) n,
$$

which imply (6).

$(6) \Rightarrow(7)$ : Set $Q(t):=\prod_{j=0}^{n}\left(t-t_{j}\right) / \prod_{j=0}^{l}\left(t-s_{j}\right)$ in (6) and apply Lemma 1.

(7) $\Rightarrow(4)$ : The polynomial $\pi \in \Pi_{n}$ which interpolates $f q$ at $t_{0}, \ldots, t_{n}$ has the representation

$$
\begin{aligned}
\pi(t) & =\sum_{i=0}^{n}(f q)\left[t_{0}, \ldots, t_{i}\right] \prod_{j=0}^{i-1}\left(t-t_{j}\right) \\
& =\sum_{i=0}^{\nu}(f q)\left[t_{0}, \ldots, t_{i}\right] \prod_{j=0}^{i-1}\left(t-t_{j}\right) \quad(\text { by }(7)) \\
& =p(t) .
\end{aligned}
$$

Remark. (a) As $\operatorname{dim} \Pi_{k}=k+1$ and $q \in \Pi_{k}$, (5) represents a system of $k$ linear equations for $k+1$ unknowns. Therefore a nontrivial solution of (5) exists. Hence, the coefficients $\alpha_{i}, i=0(1) n$, of the barycentric representation (1) are the coefficients of the Lagrangian form of $q$ with respect to all nodes $t_{0}, \ldots, t_{n}$ :

$$
q(t)=\sum_{i=0}^{n} \alpha_{i} \prod_{j=0 ; j \neq i}^{n}\left(t-t_{j}\right) .
$$

(b) Let $r:=p / q, p \in \Pi_{m}, q \in \Pi_{k}, m+k=n$, be a rational function interpolating $f \in C^{1}[a, b]$ at $t_{0}, t_{1}, \ldots, t_{n} \in[a, b]$; assume that $q(t) \neq 0$ for any $t \in[a, b]$. Then for any $t \neq t_{i}, i=0(1) n$, the following equality is valid:

$$
f(t)-r(t)=\prod_{i=0}^{n}\left(t-t_{i}\right)(f q)\left[t_{0}, \ldots, t_{n}, t\right] / q(t) .
$$

The equation

$$
(f q)\left[t_{0}, \ldots, t_{n}, t\right]=\sum_{i=0}^{n} \alpha_{i} f\left[t_{i}, t\right]
$$

can then be used to derive error estimates for rational interpolation.

Various authors derived (5) (or variants thereof) for special bases of $\Pi_{k}$ :

Examples. (a) With $Q_{i}(t):=t^{i}, i=0(1) 2 k-1, q(t):=\sum_{i=0}^{k} \sigma_{i} t^{i},(6)$ yields:

$$
\sum_{i=0}^{k} \sigma_{i}\left(f Q_{i+j}\right)\left[t_{0}, \ldots, t_{n}\right]=0, \quad j=0(1) k-1
$$


By a well-known identity for divided differences this system can be written as

$$
\sum_{i=0}^{k} \sigma_{i}\left\{\sum_{l=0}^{n} f\left(t_{l}\right) t_{l}^{i+j} / \prod_{h=0: h \neq l}^{n}\left(t_{l}-t_{h}\right)\right\}=0, \quad j=0(1) k-1 .
$$

This form of (6) was derived by Kronecker [4] and H. Werner [12] (for more historical information see Meinguet [6, p. 158 ff.]). From a computational point of view, (*) cannot be recommended: The computation of the coefficients requires many more arithmetic operations than are necessary; furthermore, numerical instabilities may occur if divided differences are evaluated in this way.

(b) If we use the Lagrange basis of $\Pi_{k}$, i.e.,

$$
q(t):=\sum_{i=0}^{k} \lambda_{i} \prod_{j=0 ; j \neq i}^{k}\left(t-t_{j}\right)
$$

then, by Lemma $1,(5)$ reads:

$$
\sum_{i=0}^{k} \lambda_{i} f\left[t_{i}, t_{k+1}, \ldots, t_{m}, t_{j}\right]=0, \quad j=m+1(1) n .
$$

In the case $k=m,(* *)$ is due to Predonzan [9]. Salzer [10] recently used the Lagrange basis in a similar way, but with respect to other nodes; he furthermore represents divided differences just like $(*)$, hence the final remarks of (a) apply to his approach, too.

(c) If we set $q(t):=\sum_{i=0}^{k} \nu_{i} \prod_{j=0}^{i-1}\left(t-t_{j}\right)$, then (5) yields

$$
\sum_{i=0}^{k} \nu_{i} f\left[t_{i}, \ldots, t_{m}, t_{j}\right]=0, \quad j=m+1(1) n,
$$

whereas (6) implies (set $Q_{j}(t):=\prod_{i=j+1}^{n}\left(t-t_{i}\right)$, so that $(f q)\left[t_{0}, \ldots, t_{j}\right]=0, j=$ $m+1(1) n)$ :

$$
\sum_{i=0}^{k} \nu_{i} f\left[t_{i}, \ldots, t_{j}\right]=0, \quad j=m+1(1) n .
$$

(10) was also derived by Opitz [8].

Since the solution of (5) may not be unique, the following notion is introduced:

Definition 3. A nontrivial solution $q \in \Pi_{k}$ of (5) will be called the "minimum degree solution" if there is no nontrivial solution of (5) of less degree; the degree of the minimum degree solution is denoted by $\delta$.

Obviously, the minimum degree solution is unique up to a (nonzero) constant multiple:

Assume that there are two nontrivial solutions $q_{1}, q_{2}$ of minimal degree; then there is an $\alpha \in \mathbf{R}$ such that $\operatorname{deg}\left(q_{1}-\alpha q_{2}\right)<\operatorname{deg}\left(q_{1}\right)$. Obviously, $q_{1}-\alpha q_{2}$ is a solution of (5), too; as (by assumption) $q_{i}, i=1,2$, are solutions of minimal degree, $q_{1}-\alpha q_{2}$ must be the trivial solution. This justifies

Definition 4. The minimum degree solution of (5) which has leading coefficient 1 will be called $q_{\delta}$ in the sequel; the corresponding polynomial of degree at most $m$ that interpolates $f q_{\delta}$ at $t_{0}, \ldots, t_{n}$ is denoted by $p_{\delta}$. 
The main properties of the minimum degree solution $q_{\delta}$ are summarized in the following

Proposition 5. (a) If $q \in \Pi_{k}$ is any solution of (5), $p \in \Pi_{m}$ the corresponding polynomial interpolating fq at $t_{0}, \ldots, t_{n}$, then there exists a polynomial $Q \in \Pi_{\operatorname{deg}(q)-\delta}$ such that $q=Q q_{\delta}$ and $p=Q p_{\delta}$.

(b) If $q_{\delta}(\tau)=0$ for some $\tau \in \mathbf{C}$, then either

$$
p_{\delta}(\tau) \neq 0 \text { and } \quad \tau \neq t_{i}, \quad i=0(1) n
$$

or

$$
p_{\delta}(\tau)=0 \text { and } \tau=t_{i} \text { for some } i, 0 \leqslant i \leqslant n .
$$

Proof. (a) Since $q p_{\delta}-q_{\delta} p \in \Pi_{n},\left(q p_{\delta}-q_{\delta} p\right)\left(t_{i}\right)=0, i=0(1) n$, the equality

$$
q p_{\delta}=q_{\delta} p
$$

is valid; $(*)$ implies that $\operatorname{deg}(p)=\operatorname{deg}(q)+\operatorname{deg}\left(p_{\delta}\right)-\delta$. Let $Q, R$ be polynomials such that $q=Q q_{\delta}+R, \operatorname{deg}(R)<\delta$; we show that $R=0$ :

For any $\pi \in \Pi_{n-\operatorname{deg}(p)-1}$ one has

$$
\begin{aligned}
0 & =(f q \pi)\left[t_{0}, \ldots, t_{n}\right] \quad(\text { by }(6)) \\
& =\left(f q_{\delta} Q \pi\right)\left[t_{0}, \ldots, t_{n}\right]+(f R \pi)\left[t_{0}, \ldots, t_{n}\right] .
\end{aligned}
$$

As $Q \pi \in \Pi_{n-\rho-1}$, where $\rho:=\operatorname{deg}\left(p_{\delta}\right),\left(f q_{\delta} Q \pi\right)\left[t_{0}, \ldots, t_{n}\right]$ vanishes by (6); thus for any $\pi \in \Pi_{n-m-1}$ one has $(f R \pi)\left[t_{0}, \ldots, t_{n}\right]=0$.

Hence, by Proposition 2, $R$ is a solution of (5) of degree $<\delta$; this implies that $R=0$. As $\operatorname{deg}\left(Q p_{\delta}\right)=\operatorname{deg}(q)-\delta+\operatorname{deg}\left(p_{\delta}\right)=\operatorname{deg}(p)$ and $\left(Q p_{\delta}\right)\left(t_{i}\right)=(f q)\left(t_{i}\right)$, $i=0(1) n$, we conclude that $Q p_{\delta}=p$.

(b) Let $\tau \in \mathbf{R}, \tau \neq t_{i}, i=0(1) n, p(t):=p_{\delta}[t, \tau], q(t):=q_{\delta}[t, \tau]$. Now assume that $p_{\delta}(\tau)=0$; then, since $q_{\delta}(\tau)=0$ by assumption, $p$ interpolates $f q$ at $t_{0}, \ldots, t_{n}$, i.e., $q$ is a solution of (5) by Proposition 2. This contradicts the minimality of $q_{\delta}$. A similar argument applies in the case $\tau \in \mathbf{C} \backslash \mathbf{R}$ if we set $p(t):=p_{\delta}[t, \tau, \bar{\tau}]$, $q(t):=q_{\delta}[t, \tau, \bar{\tau}]$.

COROLlaRY 6. If $q_{\delta}\left(t_{j}\right)=0$ for some $j, 0 \leqslant j \leqslant n$, then $t_{j}$ is a simple zero either of $q_{\delta}$ or of $p_{\delta}$.

Proof. Assume that $q_{\delta}(t)=q(t)\left(t-t_{j}\right)^{\mu}, p_{\delta}(t)=p(t)\left(t-t_{j}\right)^{\nu}, \min (\mu, \nu)>1$. Then $p_{\nu-1}$,

$$
p_{\nu-1}(t):=p(t)\left(t-t_{j}\right)^{\nu-1}
$$

interpolates $f q_{\mu-1}$ at $t_{0}, \ldots, t_{n}$, where

$$
q_{\mu-1}(t):=q(t)\left(t-t_{j}\right)^{\mu-1}
$$

this contradicts the definition of $\delta$.

Remark. Proposition 5 and Corollary 6 are essentially due to Maehly-Witzgall [5]; these results are also contained in Werner-Schaback [11, Satz 5.1] and in Wuytack [17, Lemma 2]. These authors use a different terminology, however; we therefore gave the proofs for the reader's convenience in our notation. 
Any real root $\tau$ of $q_{\delta}$ is by Proposition 5 either a pole of $r$ or $\tau=t_{j}$ for some $j$ and $\left(t_{j}, f_{j}\right)$ is an unattainable point; a point $\left(t_{j}, f_{j}\right)$ is called an unattainable point of a rational interpolation problem, if $p\left(t_{i}\right)=f_{i} q\left(t_{i}\right)$ for $i=0(1) n$, but $r\left(t_{j}\right):=$ ${ }^{*} \lim _{t \rightarrow t_{j}} p(t) / q(t) \neq f_{j}$. Since the coefficients $\alpha_{i}, i=0(1) n$, of the barycentric representation of $r$ are the coefficients of the Lagrangian representation of $q$, we can give the following simple characterization of unattainable points:

COROllary 7. A point $\left(t_{j}, f_{j}\right)$ is an unattainable point if and only if $\alpha_{j}=0$.

The possible appearance of poles of the interpolating function $r$ in the interval containing the nodes $t_{i}, i=0(1) n$, is usually considered as the most serious disadvantage of rational interpolation. None of the existing devices for rational interpolation admits any easily available criterion concerning the existence and location of poles of $r$; if, however, $r$ is represented in barycentric form, then the following result is valid:

Proposition 8. Let $t_{0}<t_{1}<\cdots<t_{n}, q_{\delta}(t)=\sum_{i=0}^{n} \alpha_{i} \prod_{j=0 ; j \neq i}^{n}\left(t-t_{j}\right), \alpha_{i} \neq 0$ $(i=0(1) n)$.

(a) If $r$ has no poles in $\left[t_{0}, t_{n}\right]$ then

$$
\operatorname{sign} \alpha_{i}=-\operatorname{sign} \alpha_{i+1}, \quad i=0(1) n-1
$$

(b) If $\operatorname{sign} \alpha_{j}=\operatorname{sign} \alpha_{j+1}$ for some $j, 0 \leqslant j \leqslant n-1$, then $r$ has an odd number of poles in $\left(t_{j}, t_{j+1}\right)$ (with their multiplicity taken into account).

The proof follows immediately from the representation

$$
\alpha_{i}=q_{\delta}\left(t_{i}\right) / \prod_{j=0: j \neq i}^{n}\left(t_{i}-t_{j}\right), \quad i=0(1) n
$$

and the fact that zeros of $q_{\delta}$ are (by Proposition 5) necessarily poles of $r$.

Remarks. (a) Proposition 8 can easily be generalized to the case where unattainable points occur.

(b) If $\delta \leqslant 1$ then the condition (11) also implies that $r$ has no pole in $\left[t_{0}, t_{n}\right]$. It is not hard to show that in this case (11) is equivalent to the condition

$$
\operatorname{sign} f\left[t_{1}, \ldots, t_{n}\right]=\operatorname{sign} f\left[t_{0}, \ldots, t_{n-1}\right]
$$

which was previously given by $\mathrm{H}$. Werner (cf. [12, Satz 3.1], [11, Satz 5.2]). In the case $\delta>1$, there may be an even number of poles in some interval $\left(t_{j}, t_{j+1}\right)$, even though (11) is fulfilled.

The coefficients of the barycentric representation (1) of $r$ thus give a great deal of information concerning poles if they are the coefficients of the Lagrangian representation of the minimum degree solution $q_{\delta}$. If the coefficients $\alpha_{i}, i=0(1) n$, are computed from any other solution $q$ of (5) of degree $>\delta$, then $r$ is not changed; the real zeros of $q$ then, however, are not necessarily poles of $r$. It is therefore important to compute the minimum degree solution $q_{\delta}$ of (5); for certain bases of $\Pi_{k}$ this is 
made possible by the following

Lemma 9. Let $p_{j} \in \Pi_{k}, \operatorname{deg}\left(p_{j}\right)=j, j=0(1) k ;$ for $d=0(1) k$ let $A_{m, d} \in \mathbf{R}^{k, d+1}$ be the matrix

$$
\left(\begin{array}{crcc}
\left(f p_{0}\right)\left[t_{0}, \ldots, t_{m}, t_{m+1}\right] & \left(f p_{1}\right)\left[t_{0}, \ldots, t_{m}, t_{m+1}\right] & \cdots & \left(f p_{d}\right)\left[t_{0}, \ldots, t_{m}, t_{m+1}\right] \\
\left(f p_{0}\right)\left[t_{0}, \ldots, t_{m}, t_{m+2}\right] & \left(f p_{1}\right)\left[t_{0}, \ldots, t_{m}, t_{m+2}\right] & \cdots & \left(f p_{d}\right)\left[t_{0}, \ldots, t_{m}, t_{m+2}\right] \\
\cdot & \cdot & \cdot & \\
\cdot & \cdot & \cdot & \\
\left(f p_{0}\right)\left[t_{0}, \ldots, t_{m}, t_{m+k}\right] & \left(f p_{1}\right)\left[t_{0}, \ldots, t_{m}, t_{m+k}\right] & \cdots & \left(f p_{d}\right)\left[t_{0}, \ldots, t_{m}, t_{m+k}\right]
\end{array}\right)
$$

Then $\delta$ is the minimal number such that $\operatorname{rank}\left(A_{m, \delta}\right)=\delta$.

Proof. Note that $A_{m, k}$ describes the matrix of the system (5) for the basis $p_{0}$, $p_{1}, \ldots, p_{k}$ of $\Pi_{k}$. If the columns of $A_{m, d}$ are linearly independent then there exists no nontrivial solution of the equation

$$
A_{m, k} \gamma=0, \quad \gamma:=\left(\gamma_{0}, \gamma_{1}, \ldots, \gamma_{k}\right) \text {, }
$$

such that $\gamma_{d+1}=\cdots=\gamma_{k}=0$; hence $\delta$ can be characterized by the condition: $\delta=\operatorname{rank} A_{m, \delta}$.

COROllary 10. If Gauss elimination with partial pivoting is applied to $A_{m, k}$ then either $\delta=k$, or $\delta$ is the index of the first column where no nonzero pivot element exists (if the columns of $A_{m, k}$ are enumerated from 0 to $k$ ).

Remark. Corollary 10 suggests a very simple strategy for the computation of the minimum degree solution $q_{\delta}$ : apply Gauss elimination with partial pivoting to the linear system (9) (or alternatively to (10)), determine $\delta$ and set $\nu_{\delta}:=1, \nu_{\delta+1}=\cdots$ $=\nu_{k}=0$. The required coefficients of $q_{\delta}$ can then easily be computed by back substitution. The exceptional case $\delta<k$ therefore requires less computations than the usual case $\delta=k$.

3. Computation of a Rational $(m, k)$-Interpolant. The algorithm for the solution of the rational interpolation problem which is motivated by the foregoing considerations consists of three steps:

Step 1. Compute the elements of the matrix $A_{m, k}$ of the linear system (5) for some basis of $\Pi_{k}$; in view of Lemma 9 and Corollary 10 we use a Newton basis with respect to the nodes $t_{0}, \ldots, t_{k}$ (cf. (9), (10)).

Step 2. Apply Gauss elimination with partial pivoting to the matrix $A_{m, k}$ in order to find the nontrivial minimum degree solution $q_{\delta}$.

Step 3. Use algorithm (2.5) of [16] to compute the coefficients $\alpha_{i}, i=0(1) n$, of the barycentric representation of $r$.

For the reader's convenience we describe Step 1 and Step 3 in more detail now. Description of Step 1. The matrix $A_{m, k}$ of the system (9) is

$$
\left(\begin{array}{cccc}
f\left[t_{0}, \ldots, t_{m}, t_{m+1}\right] & f\left[t_{1}, \ldots, t_{m}, t_{m+1}\right] & \ldots & f\left[t_{k}, \ldots, t_{m}, t_{m+1}\right] \\
f\left[t_{0}, \ldots, t_{m}, t_{m+2}\right] & f\left[t_{1}, \ldots, t_{m}, t_{m+2}\right] & \ldots & f\left[t_{k}, \ldots, t_{m}, t_{m+2}\right] \\
\cdot & \vdots & & \cdot \\
\cdot & \cdot & & \cdot \\
f\left[t_{0}, \ldots, t_{m}, t_{m+k}\right] & f\left[t_{1}, \ldots, t_{m}, t_{m+k}\right] & \ldots & f\left[t_{k}, \ldots, t_{m}, t_{m+k}\right]
\end{array}\right) .
$$


This matrix can be computed as follows:

(i) Compute the vector $v \in \mathbf{R}^{m+1}, v_{i}:=f\left[t_{i}, \ldots, t_{m}\right](i=m(-1) 0)$, by the algorithm of divided differences.

(ii) Let

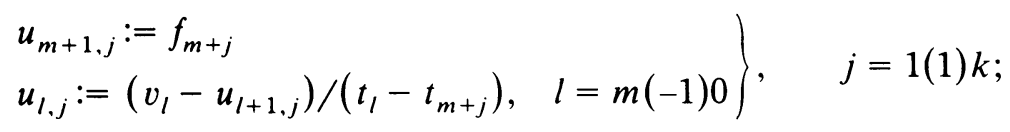

then obviously $u_{l, j}=f\left[t_{l}, \ldots, t_{m}, t_{m+j}\right], l=0(1) m, j=1(1) k$, so that

$$
\left(A_{m, k}\right)_{j i}=u_{i, j}, \quad i=0(1) k, j=1(1) k .
$$

Remarks. (a) Note that a permutation of the rows of $A_{m, k}$ corresponds to a permutation of the nodes $t_{m+1}, \ldots, t_{n}$.

(b) One may use the matrix of the linear system (10) in Step 1 as well; the computation of the elements of this matrix requires more arithmetic operations, however. This choice is preferable if several $(m, k)$-interpolants satisfying $m \geqslant k$, $m+k=n$, are to be computed. We will discuss this matter briefly at the end of this section.

Description of Step 3. Step 2 (which is routine and therefore needs no further comment) yields the minimum degree solution $q_{\delta}$ in Newtonian form:

$$
q_{\delta}(t)=\sum_{i=0}^{\delta} \nu_{i} \prod_{j=0}^{i-1}\left(t-t_{j}\right), \quad \nu_{\delta}=1 .
$$

As already mentioned, the required coefficients $\alpha_{i}, i=0(1) n$, of (1) are the coefficients of the Lagrangian representation of $q_{\delta}$ with respect to all nodes $t_{0}, \ldots, t_{n}$. Therefore they can be computed efficiently with algorithm (2.5) of [16]:

Set

$$
\begin{aligned}
& a_{i, 0}:=\nu_{i}, \quad i=0(1) \delta, \quad a_{i, 0}:=0, \quad i=\delta+1(1) n \\
& \left.\begin{array}{l}
a_{j, i}:=a_{j, i-1} /\left(t_{j}-t_{i}\right) \\
a_{i, j+1}:=a_{i, j}-a_{j, i}
\end{array}\right\}, \quad j=0(1) i-1, i=1(1) n
\end{aligned}
$$

then $\alpha_{i}=a_{i, n}, i=0(1) n$.

If, for fixed $m$ and $k$, several rational $(m, k)$-interpolants must be computed for different sets of data, but fixed nodes $t_{0}, \ldots, t_{n}$, then the computations should proceed as follows:

Set $a_{i, 0}:=\nu_{i}, i=0(1) \delta, a_{i, 0}:=0, i=\delta+1(1) k$; if

$$
\left.\begin{array}{l}
a_{j, i}:=a_{j, i-1} /\left(t_{j}-t_{i}\right) \\
a_{i, j+1}:=a_{i, j}-a_{j, i}
\end{array}\right\}, \quad j=0(1) i-1, i=1(1) k,
$$

then $q_{\delta}(t)=\sum_{i=0}^{k} a_{i, k} \prod_{j=0 ; j \neq i}^{k}\left(t-t_{j}\right)$.

From $\alpha_{i}=q_{\delta}\left(t_{i}\right) / \prod_{j=0 ; j \neq i}^{n}\left(t_{i}-t_{j}\right), i=0(1) n$, we conclude that

$$
\begin{gathered}
\alpha_{l}=a_{l, k} / \prod_{j=k+1}^{n}\left(t_{l}-t_{j}\right), \quad l=0(1) k, \\
\alpha_{l}=\left(\sum_{i=0}^{k} a_{i, k} /\left(t_{l}-t_{i}\right)\right) / \prod_{j=k+1 ; j \neq l}^{n}\left(t_{l}-t_{j}\right), \quad l=k+1(1) n .
\end{gathered}
$$


The quantities $1 / \prod_{j=k+1}^{n}\left(t_{l}-t_{j}\right), \quad l=0(1) k$, and $1 / \prod_{j=k+1 ; j \neq l}^{n}\left(t_{l}-t_{j}\right), \quad l=$ $k+1(1) n$ (which can be computed efficiently by algorithm (2.5) of [16], too!) may be computed once and for all; they can be computed analytically in the case of equidistant nodes.

Remark. If one is interested in various rational $(m, k)$-interpolants (with $m \geqslant$ $k, m+k=n$ ) then one should proceed as follows:

Step 1. Compute the matrix $A_{m, k}$ of (10):

$$
\left(\begin{array}{cccc}
f\left[t_{0}, \ldots, t_{m+k}\right] & \ldots & f\left[t_{k-1}, \ldots, t_{m+k}\right] & f\left[t_{k}, \ldots, t_{m+k}\right] \\
\cdot & & \cdot & \cdot \\
\cdot & & \cdot & \cdot \\
f\left[t_{0}, \ldots, t_{m+2}\right] & \ldots & f\left[t_{k-1}, \ldots, t_{m+2}\right] & f\left[t_{k}, \ldots, t_{m+2}\right] \\
f\left[t_{0}, \ldots, t_{m+1}\right] & \ldots & f\left[t_{k-1}, \ldots, t_{m+1}\right] & f\left[t_{k}, \ldots, t_{m+1}\right]
\end{array}\right)
$$

using the algorithm of divided differences. Note that the $j \times(j+1)$ principal submatrix is identical with $A_{n-j, j}, j=1(1) k$.

Steps 2,3. Apply Gauss elimination without pivoting (assuming that this is possible) to $A_{m, k}$; it is then easy to compute all $(n-j, j)$-interpolants of $f$ from the corresponding $j \times(j+1)$ principal submatrices, $j=1(1) k$.

Numerical Examples. In the following examples we normalize the coefficients $\alpha_{i}$, $i=0(1) n$, such that

$$
\sum_{i=0}^{n}\left|\alpha_{i}\right|=1
$$

(a) From our point of view it is the major advantage of our representation (1) of the interpolating rational function $r$ that it contains a good deal of information on the existence and location of poles. This is demonstrated in an example considered by $\mathrm{H}$. Werner [15, Example 6]. It is very instructive to compare the graphs of the rational functions given in [15] with our results: all poles are easily detected by the sign pattern of the coefficients $\alpha_{i}, i=0(1) 8$, which are displayed in Table 1 .

TABLE 1

Coefficients $\boldsymbol{\alpha}_{i}$ for Example (a)

\begin{tabular}{rrrrrr}
\hline$t_{i}$ & $f_{i}$ & \multicolumn{4}{c}{$\alpha_{i}$ for $(m, k)=$} \\
& & \multicolumn{1}{c}{$(4,4)$} & \multicolumn{1}{c}{$(5,3)$} & \multicolumn{1}{c}{$(6,2)$} & \multicolumn{1}{c}{$(7,1)$} \\
\hline 0 & -2 & $3.0000 \mathrm{E}-2$ & $-6.4202 \mathrm{E}-2$ & $2.3544 \mathrm{E}-2$ & $-1.3194 \mathrm{E}-2$ \\
1 & -1 & $-1.0000 \mathrm{E}-1$ & $1.7899 \mathrm{E}-1$ & $-9.0753 \mathrm{E}-2$ & $8.5532 \mathrm{E}-2$ \\
2 & 0 & $9.0000 \mathrm{E}-2$ & $-3.5019 \mathrm{E}-2$ & $8.8185 \mathrm{E}-2$ & $-2.2930 \mathrm{E}-1$ \\
3 & 0 & $4.0000 \mathrm{E}-2$ & $-2.9572 \mathrm{E}-1$ & $5.8219 \mathrm{E}-2$ & $3.1847 \mathrm{E}-1$ \\
4 & 0 & $-6.0000 \mathrm{E}-2$ & $2.3346 \mathrm{E}-1$ & $-8.5616 \mathrm{E}-2$ & $-2.2293 \mathrm{E}-1$ \\
5 & 1 & $-1.4000 \mathrm{E}-1$ & $5.4474 \mathrm{E}-2$ & $-1.4555 \mathrm{E}-2$ & $3.8216 \mathrm{E}-2$ \\
6 & 0 & $2.9000 \mathrm{E}-1$ & $-4.2802 \mathrm{E}-2$ & $2.9195 \mathrm{E}-1$ & $5.0955 \mathrm{E}-2$ \\
7 & -1 & $-2.0000 \mathrm{E}-1$ & $-6.2257 \mathrm{E}-2$ & $-1.7808 \mathrm{E}-1$ & $-3.4577 \mathrm{E}-2$ \\
8 & -2 & $5.0000 \mathrm{E}-2$ & $3.3074 \mathrm{E}-2$ & $3.8099 \mathrm{E}-2$ & $6.8244 \mathrm{E}-3$ \\
\hline
\end{tabular}


It is, e.g., evident from these data that the rational (4,4)-interpolant has at least one pole in the intervals $(2,3)$ and $(4,5)$; actually both intervals contain exactly one pole.

(b) In the next example there occurs an unattainable point: Interpolate the data $(0,1),(2,2),(2.5,9.5),(3,2.5),(4,3)$ by a rational function such that $m=3, k=1$ (cf. H. Werner [15, Example 4]). The computed coefficients are shown in Table 2.

TABLE 2

Coefficents $\alpha_{i}$ for Example (b)

\begin{tabular}{lc}
\hline$t_{i}$ & $\alpha_{i}$ \\
\hline 0 & $-5.5555 \mathrm{E}-2$ \\
2 & $3.3333 \mathrm{E}-1$ \\
2.5 & $5.7824 \mathrm{E}-19$ \\
3 & $-4.4444 \mathrm{E}-1$ \\
4 & $1.6666 \mathrm{E}-1$ \\
\hline
\end{tabular}

It is evident that (within machine precision: 63 binary digits) the point $(2.5,9.5)$ is an unattainable point of the problem.

(c) We interpolate $f(t)=t^{4}$ at $t_{i}:=5 i / 6, i=0(1) 5$ (cf. H. Werner [14, p. 332]); for $m=4, k=1$ the denominator $q$ was computed as

$$
q(t)=t-1.60128 \mathrm{E}+18
$$

The normalized coefficients $\alpha_{i}, i=0(1) 5$, as computed, are shown in Table 3.

TABLE 3

Coefficients $\alpha_{i}$ for Example (c)

\begin{tabular}{rr}
\hline$i$ & \multicolumn{1}{c}{$\alpha_{i}$} \\
\hline 0 & $-3.1250 \mathrm{E}-2$ \\
1 & $1.5625 \mathrm{E}-1$ \\
2 & $-3.1250 \mathrm{E}-1$ \\
3 & $3.1250 \mathrm{E}-1$ \\
4 & $-1.5625 \mathrm{E}-1$ \\
5 & $3.1250 \mathrm{E}-2$ \\
\hline
\end{tabular}

Within machine precision one gets exactly the same normalized coefficients for the correct denominator $q(t)=1$; i.e., the fact that a pivot element which should be zero in exact arithmetic was not detected had no influence on the final result. This effect is reflected by the fact that the computed denominator has a coefficient of size 1 /eps, where eps denotes the machine precision.

We emphasize, however, that sometimes rounding errors may cause troubles: If in the course of Gauss' algorithm a row is encountered all of whose entries were zero in exact arithmetic but are nonzero due to roundoff and if this fact is overlooked, then the algorithm produces an "innocent looking" denominator which has no minimum degree; it then cannot be ruled out that the computed denominator has a root which 
is not a pole of the interpolant. Thus, if small pivot elements occur, conclusions concerning the existence and location of poles of the interpolant must be drawn with caution; the interpolation property of the computed rational function is not affected by these effects as long as the computed coefficients remain nonzero.

4. Differentiation of Rational Functions. A distinct advantage of the barycentric represention of rational function, which appears to have been overlooked so far (even if (1) represents a polynomial), is the fact that this form of the rational function admits a very simple formula for its derivatives. Usually, formulas for the derivatives of a rational function are based on partial fraction decomposition, which requires knowledge of the zeros of the denominator (cf. Henrici [3, p. $569 \mathrm{ff}$.]).

Proposition 11. Let $r$ be a rational function given in its barycentric form (1), with $\alpha_{i} \neq 0, i=0(1) n$. Assume that $\xi$ is not a pole of $r$, then

$$
r^{(k)}(\xi) / k !=\sum_{i=0}^{n} \frac{\alpha_{i}}{\xi-t_{i}} r\left[(\xi)^{k}, t_{i}\right] / \sum_{i=0}^{n} \frac{\alpha_{i}}{\xi-t_{i}}
$$

$$
\text { if } \xi \neq t_{i}, i=0(1) n, k \geqslant 0 .
$$

$$
r^{(k)}\left(t_{j}\right) / k !=-\left(\sum_{i=0 ; i \neq j}^{n} \alpha_{i} r\left[\left(t_{j}\right)^{k}, t_{i}\right]\right) / \alpha_{j}, \quad 0 \leqslant j \leqslant n, k \geqslant 1 .
$$

(Here, and in the sequel, we use the notation $r\left[(\xi)^{k}, t\right]$ instead of $r[\xi, \ldots, \xi, t]$ for a $k$-fold argument.)

Proof. (12) is evident for $k=0$; now assume that (12) is true for some $k \geqslant 0$. Then (12) implies

$$
\sum_{i=0}^{n} \alpha_{i} r\left[(\xi)^{k+1}, t_{i}\right]=0
$$

differentiation with respect to $\xi$ yields

$$
(k+1) \sum_{i=0}^{n} \alpha_{i} r\left[(\xi)^{k+2}, t_{i}\right]=0 .
$$

If $\xi \neq t_{j}, j=0(1) n$, then from

$$
0=\sum_{i=0}^{n} \alpha_{i} r\left[(\xi)^{k+2}, t_{i}\right]=\sum_{i=0}^{n} \alpha_{i}\left\{r\left[(\xi)^{k+1}, t_{i}\right]-r\left[(\xi)^{k+2}\right]\right\} /\left(t_{i}-\xi\right)
$$

it follows that

$$
\begin{aligned}
r\left[(\xi)^{k+2}\right] & =r^{(k+1)}(\xi) /(k+1) ! \\
& =\sum_{i=0}^{n} \frac{\alpha_{i}}{\xi-t_{i}} r\left[(\xi)^{k+1}, t_{i}\right] / \sum_{i=0}^{n} \frac{\alpha_{i}}{\xi-t_{i}},
\end{aligned}
$$

i.e., (12) is true for $k+1$; the proof of (13) is similar.

Remark. There is a remarkable analogy between (12) and Cauchy's integral formula

$$
n(\Gamma, \zeta) f^{(k)}(\zeta) / k !=\oint_{\Gamma} f(z) /(z-\zeta)^{k+1} d z / 2 \pi i
$$


where $f$ is an analytic function defined in a simply connected domain $R \subseteq \mathbf{C}, \Gamma$ a piecewise regular closed curve not passing through $\zeta, n(\Gamma, \zeta)$ the winding number of $\zeta$ with respect to $\Gamma \subseteq R$ (cf., e.g., Henrici [3, p. 245 ff.]). Using the identities

$$
\begin{gathered}
\oint_{\Gamma} 1 /(z-\zeta) d z=2 \pi i n(\Gamma, \zeta) \\
\oint_{\Gamma} f\left[(\zeta)^{k}, z\right] /(z-\zeta) d z=\oint_{\Gamma} f(z) /(z-\zeta)^{k+1} d z
\end{gathered}
$$

(14) may be written in the form

$$
f^{(k)}(\zeta) / k !=\oint_{\Gamma} \frac{f\left[(\zeta)^{k}, z\right]}{z-\zeta} d z / \oint_{\Gamma} \frac{1}{z-\zeta} d z \quad \text { if } n(\Gamma, \zeta) \neq 0 .
$$

Thus (12) may be interpreted as a discrete analogue of the Cauchy integral formula (14) for rational functions; note that (12) remains valid if the nodes $t_{i}, i=0(1) n$, are complex.

Proposition 11 gives rise to a simple scheme for the recursive computation of the derivatives of a rational function:

Proposition 12. Let $r$ be a rational function given in its barycentric form (1), with $\alpha_{i} \neq 0, i=0(1) n$; assume that $\xi$ is not a pole of $r$.

(a) If $\xi \neq t_{i}, i=0(1) n$, let

$$
\begin{gathered}
\gamma_{i}:=\frac{\alpha_{i}}{\xi-t_{i}} / \sum_{j=0}^{n} \frac{\alpha_{j}}{\xi-t_{j}}, \quad i=0(1) n, \\
\delta_{i, 0}:=f_{i}, \quad i=0(1) n, \\
\left.\begin{array}{c}
\varphi_{k}:=\sum_{i=0}^{n} \gamma_{i} \delta_{i, k} \\
\delta_{i, k+1}:=\left(\delta_{i, k}-\varphi_{k}\right) /\left(t_{i}-\xi\right)
\end{array}\right\}, \quad k=0,1,2,3, \ldots
\end{gathered}
$$

Then $\varphi_{k}=r^{(k)}(\xi) / k !, \delta_{i, k}=r\left[(\xi)^{k}, t_{i}\right], i=0(1) n, k=0,1,2,3, \ldots$

(b) Let $\xi=t_{j}$; if

$$
\begin{aligned}
& \gamma_{i}:=-\alpha_{i} / \alpha_{j}, \quad i=0(1) j-1, j+1(1) n, \\
& \delta_{i, 1}:=\left(f_{i}-f_{j}\right) /\left(t_{i}-t_{j}\right), \quad i=0(1) j-1, j+1(1) n, \\
& \left.\begin{array}{l}
\varphi_{k}:=\sum_{i=0: i \neq j}^{n} \gamma_{i} \delta_{i, k} \\
\delta_{i, k+1}:=\left(\delta_{i, k}-\varphi_{k}\right) /\left(t_{i}-t_{j}\right), \quad i=0(1) j-1, j+1(1) n
\end{array}\right\}, \quad k=1,2,3, \ldots, \\
& \text { then } \varphi_{k}=r^{(k)}\left(t_{j}\right) / k !, \delta_{i, k}=r\left[\left(t_{j}\right)^{k}, t_{i}\right], i=0(1) j-1, j+1(1) n, k=1,2,3, \ldots
\end{aligned}
$$

The proof follows from Proposition 11 by induction.

Proposition 11 may also be used to derive formulas for numerical differentiation based on rational interpolation; for this purpose it is, however, more convenient to use another representation of $r^{\prime}\left(t_{j}\right), j=0(1) n$ :

Proposition 13. Let $P \in \Pi_{n}$ be the unique polynomial interpolating $f \in C^{1}[a, b]$ at $t_{0}, \ldots, t_{n} \in[a, b]$; assume that $q$ is a nontrivial solution of (5) such that $q\left(t_{i}\right) \neq 0$, 
$i=0(1) n, r=p / q, p \in \Pi_{m}$. Furthermore, let $Q_{j}(t):=q\left[t, t_{j}\right]$. Then

$$
r^{\prime}\left(t_{j}\right)=P^{\prime}\left(t_{j}\right)-\prod_{i=0 ; i \neq j}^{n}\left(t_{j}-t_{i}\right) \frac{\left(f Q_{j}\right)\left[t_{0}, \ldots, t_{n}\right]}{q\left(t_{j}\right)}, \quad j=0(1) n \text {. }
$$

Proof. From the equation

$$
f(t)-r(t)=\prod_{i=0}^{n}\left(t-t_{i}\right)(f q)\left[t_{0}, \ldots, t_{n}, t\right] / q(t)
$$

one deduces

$$
f^{\prime}\left(t_{j}\right)-r^{\prime}\left(t_{j}\right)=\prod_{i=0 ; i \neq j}^{n}\left(t_{j}-t_{i}\right) \frac{(f q)\left[t_{0}, \ldots, t_{n}, t_{j}\right]}{q\left(t_{j}\right)}
$$

and

$$
f^{\prime}\left(t_{j}\right)-P^{\prime}\left(t_{j}\right)=\prod_{i=0: i \neq j}^{n}\left(t_{j}-t_{i}\right) f\left[t_{0}, \ldots, t_{n}, t_{j}\right]
$$

so that

$$
\begin{aligned}
r^{\prime}\left(t_{j}\right)-P^{\prime}\left(t_{j}\right) & =\prod_{i=0: i \neq j}^{n}\left(t_{j}-t_{i}\right)\left\{f\left[t_{0}, \ldots, t_{n}, t_{j}\right]-\frac{(f q)\left[t_{0}, \ldots, t_{n}, t_{j}\right]}{q\left(t_{j}\right)}\right\} \\
& =\prod_{i=0 ; i \neq j}^{n}\left(t_{j}-t_{i}\right) \frac{\left(f\left(q\left(t_{j}\right)-q\right)\right)\left[t_{0}, \ldots, t_{n}, t_{j}\right]}{q\left(t_{j}\right)} .
\end{aligned}
$$

(16) now follows from Lemma 1.

Corollary 14. If $k=1, m=n-1$ in Proposition 13, then

$$
\begin{aligned}
& r^{\prime}\left(t_{j}\right)=P^{\prime}\left(t_{j}\right)+\prod_{i=0 ; i \neq j}^{n}\left(t_{j}-t_{i}\right) \frac{f\left[t_{0}, \ldots, t_{n}\right]^{2}}{f\left[t_{0}, \ldots, t_{j-1}, t_{j+1}, \ldots, t_{n}\right]}, \\
& j=0(1) n .
\end{aligned}
$$

Proof. From (5) one readily concludes that

$$
q(t)=f\left[t_{0}, \ldots, t_{n}\right]\left(t-t_{j}\right)-f\left[t_{0}, \ldots, t_{j-1}, t_{j+1}, \ldots, t_{n}\right]
$$

the assertion therefore follows from Lemma 1 and (16).

Remarks. (a) Note that the value $P^{\prime}\left(t_{j}\right)$ in (16), (17) represents the result of a usual differentiation formula based on polynomial interpolation of the data $\left(t_{0}, f_{0}\right), \ldots,\left(t_{n}, f_{n}\right)$; the second term on the right side of (16) (resp. (17)) thus is a correction which is due to the fact that rational interpolation is used instead of polynomial interpolation.

(b) Differentiation formulas based on rational interpolation may be useful if "nonpolynomial information" concerning the function $f$, which is to be differentiated, is known; e.g., if it is known that near the point where $f$ is to be differentiated there is a pole, then one cannot exploit this additional information in usual differentiation formulas based on polynomial interpolation.

Examples. (a) Let $f(t)=\tan (t)$ be given at $t_{0}=1.3, t_{1}=1.4, t_{2}=1.5$; if the derivative of $f$ at the nodes is to be approximated from these data then (with the notations of Corollary 14) formula (17) yields the results in Table 4. 
TABLE 4

Numerical differentiation of the tangent function

\begin{tabular}{crrr}
\hline$t_{j}$ & $f^{\prime}\left(t_{j}\right)$ & \multicolumn{1}{c}{$P^{\prime}\left(t_{j}\right)$} & \multicolumn{1}{c}{$r^{\prime}\left(t_{j}\right)$} \\
\hline 1.3 & 13.975 & -8.581 & 13.882 \\
1.4 & 34.615 & 52.497 & 34.731 \\
1.5 & 199.850 & 113.574 & 198.520
\end{tabular}

Unlike the differentiation formula based on polynomial interpolation (cf. the third column), our differentiation formula (17) gives quite satisfactory results.

(b) Let $f(t)=\arctan (t)$ be given at $t_{0}=1, t_{1}=2, t_{2}=3$; with these data the differentiation formula (17) yields the results in Table 5.

TABLE 5

Numerical differentiation of the arctangent function

\begin{tabular}{cccc}
\hline$t_{j}$ & $f^{\prime}\left(t_{j}\right)$ & $P^{\prime}\left(t_{j}\right)$ & $r^{\prime}\left(t_{j}\right)$ \\
\hline 1 & 0.5 & 0.412 & 0.526 \\
2 & 0.2 & 0.232 & 0.197 \\
3 & 0.1 & 0.052 & 0.102 \\
\hline
\end{tabular}

Both $f$ and its $(1,1)$-interpolant $r$ share the common property that they are asymptotically constant. As the stepsize (which is 1 in the present example) is large, differentiation formulas based on polynomial interpolation give poor results.

Fachbereich Mathematik

der Johannes Gutenberg-Universität

D 65 Mainz, Federal Republic of Germany

DFVLR

German Space Operations Center

D 8031 Oberpfaffenhofen, Federal Republic of Germany

1. A.- L. Cauchy, "Sur la formule de Lagrange relative à l'interpolation," Cours d'Analyse de l'Ecole Royale Polytéchnique (Analyse Algébrique), Note V, Paris, 1821, pp. 429-433, reprinted in: Oeuvres, sér. 2, Vol. III, 1897.

2. P. R. Graves-Morris, "Efficient reliable rational interpolation," Padé Approximation and its Applications (Amsterdam, 1980) (M. G. de Bruin, H. v. Rossum, eds.), Lecture Notes in Math., vol. 888, Springer, Berlin, 1981, pp. 28-63.

3. P. Henrici, Applied and Computational Complex Analysis, Vol. 1, Wiley, New York, 1974.

4. L. KRONECKER, "Zur Theorie der Elimination einer Variablen aus zwei algebraischen Gleichungen," Monatsber. Königl. Preuss. Akad. Wiss. Berlin, 1881, pp. 535-600.

5. H. Maehly \& C. Witzgall, "Tschebyscheff-Approximationen in kleinen Intervallen. II," Numer. Math., v. 2, 1960, pp. 293-307.

6. J. Meinguet, "On the solubility of the Cauchy interpolation problem," Approximation Theory (A. Talbot, ed.), Academic Press, London, 1970, pp. 137-163.

7. L. M. Milne-Thomson, The Calculus of Finite Differences, Macmillan, London, 1933.

8. G. OpIrz, “Steigungsmatrizen," Z. Angew. Math. Mech., v. 44, 1964, pp. T52-T54.

9. A. Predonzan, "Su una formula d'interpolazione per le funzioni razionali," Rend. Sem. Mat. Univ. Padova, v. 22, 1953, pp. 417-425. 
10. H. E. Salzer, "Rational interpolation using incomplete barycentric forms," Z. Angew. Math. Mech., v. 61, 1981, pp. 161-164.

11. H. Werner \& R. Schaback, Praktische Mathematik. II, Springer, Berlin, 1972.

12. H. WERnER, "Rationale Tschebyscheff-Approximation, Eigenwerttheorie und Differenzenrechnung." Arch. Rational Mech. Anal., v. 13, 1963, pp. 330-347.

13. H. Werner, "A reliable method for rational interpolation," Pade Approximation and its Applications (L. Wuytack, ed.), Lecture Notes in Math., Vol. 765, Springer, Berlin, 1979, pp. 257-277.

14. H. Werner, "Ein Algorithmus zur rationalen Interpolation," Numerical Methods of Approximation Theory, Vol. 5 (L. Collatz, G. Meinardus, H. Werner, eds.), ISNM 52, Birkhäuser, Basel, 1980, pp. 319-337.

15. H. WERNER, "A reliable and numerically stable program for rational interpolation of Lagrange data," Computing, v. 31, 1983, pp. 269-286.

16. W. Werner, "Polynomial interpolation: Lagrange versus Newton," Math. Comp., v. 43, 1984, pp. 205-217.

17. L. WUYTACK, "On some aspects of the rational interpolation problem," SIAM J. Numer. Anal., v. 11, 1974, pp. 52-60. 